\title{
Some Corrections on My Paper "Some Connections between Heyting Valued Set Theory and Algebraic Geometry- Prolegomena to Intuitionistic Algebraic Geometry"
}

By

\author{
Hirokazu NishimURA*
}

(1) In the definition of a morphism of locally ringed cHas (p. 506), it is not $f^{\#}: \mathcal{O}_{\Omega} \rightarrow f_{*} \mathcal{O}_{H}$ but its left adjoint ${ }^{\sharp}: f^{*} \mathcal{O}_{\Omega} \rightarrow \mathcal{O}_{H}$, regarded as a homomorphism of rings in $V^{(H)}$, that should be required to be a local homomorphism of local rings. Due modification should be made wherever this notion is concerned.

(2) In the proof of Theorem 4.3 (p. 513), since the degree $\partial(f)$ of a polynomial $f$ is not available in intuitionistic algebra generally, $h_{\mathfrak{a}}$ should be defined to be the homogeneous ideal generated by the following set:

$$
\left\{x_{0}^{m+1} f\left\{x_{1} x_{0}^{-1}, \ldots, x_{n} x_{0}^{-1}\right) \mid x_{0}^{m} f\left(x_{1} x_{0}^{-1}, \ldots, x_{n} x_{0}^{-1}\right) \in A\left[x_{0}, \ldots, x_{n}\right], f \in \mathfrak{a}, m \geq 0\right\} .
$$

(3) Our definition of a local ring and that of a local homomorphism of local rings are the familiar geometric ones, for which the reader is referred, e.g., to Scott $[12 ; 3.4]$.

(4) In the definition of Čech cohomology groups $H^{n}(\Omega, \mathscr{F})$ of a $\mathrm{cHa} \Omega$ with sheaf coefficients $\mathscr{F}$ in $\S 5$ we consider only those coverings $\mathfrak{U} \subset \Omega$ such that

$$
\mathfrak{U}=\{p \in \Omega \mid \exists q \in \mathfrak{U}(p \leq q)\} .
$$

The preorder among these coverings is simply the set-theoretic inclusion and a refinement mapping for coverings $\mathfrak{B} \subset \mathfrak{U}$ is always the inclusion mapping. Thus there is no danger that the axiom of choice sneaks into our discussion.

Communicated by S. Takasu, November 2, 1987.

* Institute of Mathematics, University of Tsukuba, Ibaraki 305, Japan. 
